\title{
High-resolution imaging of depth filter structures using X-ray computed tomography
}

\author{
T. F. Johnson ${ }^{1}$ (D) F. lacoviello ${ }^{2}$, J. H. Welsh ${ }^{3}$, P. R. Shearing ${ }^{2}$, and D. G. Bracewell ${ }^{1, *}$ \\ ${ }^{1}$ Department of Biochemical Engineering, University College London, Bernard Katz, London WC1E 6BT, UK \\ ${ }^{2}$ Electrochemical Innovation Laboratory, Department of Chemical Engineering, University College London, Torrington Place, \\ London WC1E 7JE, UK \\ ${ }^{3}$ Pall Biotech, 5 Harbourgate Business Park, Southampton Road, Portsmouth PO6 4BQ, UK
}

Received: 5 May 2021

Accepted: 5 June 2021

Published online:

18 June 2021

(C) The Author(s) 2021

\begin{abstract}
A multiple length scale approach to the imaging and measurement of depth filters using X-ray computed tomography is described. Three different filter grades of varying nominal retention ratings were visualized in $3 \mathrm{D}$ and compared quantitatively based on porosity, pore size and tortuosity. Positional based analysis within the filters revealed greater voidage and average pore sizes in the upstream quartile before reducing progressively through the filter from the center to the downstream quartile, with these results visually supported by voidage distance maps in each case. Flow simulation to display tortuous paths that flow may take through internal voidage were examined.

Digital reconstructions were capable of identifying individual constituents of voidage, cellulose and perlite inside each depth filter grade, with elemental analysis on upstream and downstream surfaces confirming perlite presence. Achieving an appropriate pixel size was of particular importance when optimizing imaging conditions for all grades examined. A $3 \mu \mathrm{m}$ pixel size was capable of representing internal macropores of each filter structure; however, for the finest grade, an improvement to a $1 \mu \mathrm{m}$ pixel size was required in order to resolve micropores and small perlite shards. Enhancing the pixel size resulted in average porosity measurements of $70 \%$ to $80 \%$ for all grades.
\end{abstract}

Handling Editor: M. Grant Norton.

Address correspondence to E-mail: d.bracewell@ucl.ac.uk 


\section{GRAPHICAL ABSTRACT}

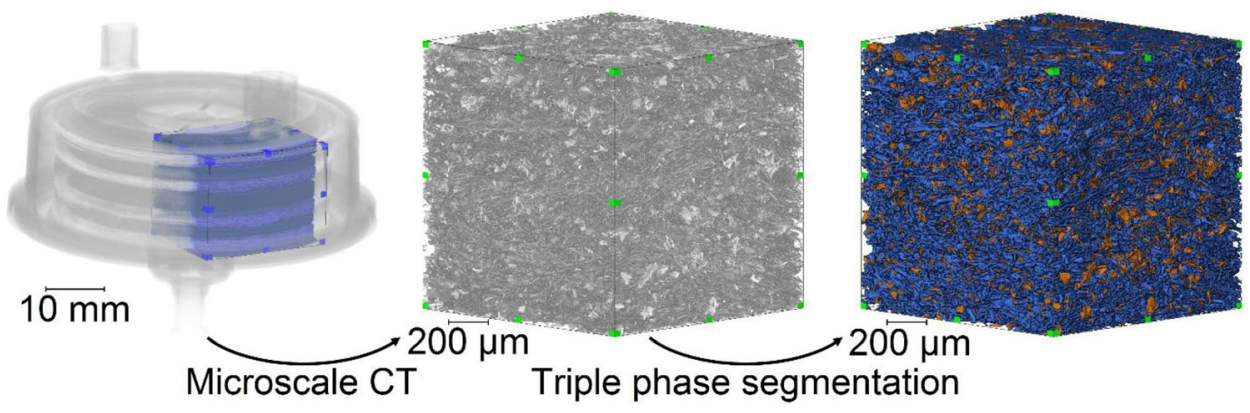

\section{Introduction}

Depth filters are commonly used early in downstream processing for various applications within the life sciences sector, for example antibody manufacturing for therapeutic purposes [1-3]. The three dimensional structure of a depth filter contains a specified range of pores in order to entrap and prevent unwanted material passing through, while enabling permeation of desired process constituents to be separated in following steps such as capture chromatography [4-6]. Clarification using depth filtration allows for cells to be retained by the structure while smaller components such as proteins pass through, with cell debris also retained if the intended product is expressed intracellularly and must be extracted through approaches such as homogenization [7-9]. Downstream processing using depth filters can also remove smaller feed constituents including host cell proteins and DNA through adsorptive interactions [10].

The media used in depth filtration often contains multiple materials of construction that include a cellulose base, perlite and diatomaceous earth [11]. A detailed, internal porous structure is formed that is essential to function and performance that has to cope with increasing feed stream titers that add additional burden to downstream bioprocessing that is an increasing challenge [12]. Advances in 3D imaging capability for multiple techniques has enabled materials to be visualized and characterized in great detail [13-17]. Three major approaches for 3D imaging materials at high resolution include serial block face (SBF) microtomy, focused ion beam (FIB) microscopy and X-ray computed tomography (CT) [18-27]. SBF and FIB microscopy are both capable of achieving nanoscale resolutions; however, they both require extensive preparation of the sample which is destroyed during the imaging process [28].

$X$-ray CT can also achieve high resolutions that are suitable for imaging structures such as depth filter pore networks while being non-invasive and nondestructive. All three techniques have been used to examine internal chromatography bead and packed bed structures in addition to multiple other material characterization applications including batteries and fuel cells [29-35]. A tomographic approach enables digitally reconstructed volumes of an imaged sample to be measured for geometric characteristics such as porosity and pore size.

In particular measuring tortuosity by applying simulation tools is of interest following 3D imaging, where using other methods can be difficult to both define and accurately evaluate this factor [36-41]. Additionally, employing a multi-length scale approach enables the capsules that the depth filter material resides within to be visualized in $3 \mathrm{D}$, again without the need for cutting open the sample which would damage or destroy the structure [42-44].

In this study, X-ray CT is applied to image the internal, 3D structure of three commercially available depth filters at a microscale resolution. Evaluating samples with multiple materials of construction enables triple-phase tomography to be explored, defined as three phases within the 3D dataset rather than just material and voidage [45]. Here, the three 
phases of interest are perlite, cellulose and voidage that require sufficient image quality and contrast to be successfully resolved and segmented.

\section{Materials and methods}

Three depth filter materials in the form of sheets or capsules were selected from Pall Biotech: a fine (nominal retention rating: 2-4 $\mu \mathrm{m}$ ), medium $(6-15 \mu \mathrm{m})$ and coarse $(11-30 \mu \mathrm{m})$ variant. Samples were obtained from sheets, with dimensions of $350 \mathrm{~mm}$ by $500 \mathrm{~mm}$ and a thickness of approximately $3 \mathrm{~mm}$. Sub-sections were isolated using a sharp scalpel with a small amount of excess material taken to avoid disturbing or damaging the intended volume of interest. Beyond physical isolation, no further preparative processing was performed to minimize the risk of changing the structure of each sample before imaging.

X-ray CT imaging was performed using a Nikon XT H 225 system (Tring, United Kingdom) at the Electrochemical Innovation Laboratory (EIL) at UCL, achieving a pixel size of $3 \mu \mathrm{m}$ in each case which was the maximum possible in reflection mode. In order to make reflection ( $3 \mu \mathrm{m}$ spot size) and transmission ( $2 \mu \mathrm{m}$ spot size) mode imaging comparable, optimization for use of a tungsten target was performed. Final operating conditions selected were a primary accelerating voltage of $125 \mathrm{keV}$, power rating no more than $7 \mathrm{~W}$, exposure time of $1 \mathrm{~s}$ and 4 frames over 3,142 projections per scan. For transmission mode imaging, the power was reduced to $3 \mathrm{~W}$ and digital gain increased fourfold.

For further resolution improvements, a ZEISS Xradia Versa 620 (Pleasanton, USA) was used on the same depth filter samples. A primary accelerating voltage of $40 \mathrm{keV}$ was applied with a $3 \mathrm{~W}$ power rating in each case over 1,601 projections, where for $1 \mu \mathrm{m}$ and $0.5 \mu \mathrm{m}$ pixel size imaging respective $4 \mathrm{X}$ and $20 \times$ (binning 2) lenses were used with a $15 \mathrm{~s}$ exposure. Versa imaging at these improved pixel sizes resulted in internal tomography being necessary as the entire thickness of each sample could not be viewed, with internal scans of the central $\sim 1 \mu \mathrm{m}$ examined in this study. Figure 1 displays a schematic for X-ray CT image acquisition in addition to an isolated sample prepared for high resolution scanning.
Reconstruction was performed in X-Tek or ZEISS software before being loaded into Thermo Fisher (MA, USA) Avizo ${ }^{\circledR}$ Fire 9.5 for processing. Raw volumes were cropped into $3 \mathrm{~mm}$ or $1.5 \mathrm{~mm}$ cubes and directional issues such as rotation were corrected. Segmentation of perlite, cellulose and voidage was performed using interactive thresholding based on raw volumes that had undergone 'non-local means' and 'unsharp masking' pre-processing, with additional post-processing required in some cases. Measurement of porosity and average pore size was based on sub-volume analysis in the upper quarter, center and lower quarter of each volume using 'volume fraction' and 'average space thickness' commands. Voidage distance maps were applied with the pore size represented through color-matching of voxels to the relative distance to the nearest material voxel.

Tortuosity factor was evaluated using the MATLAB plugin TauFactor [39] by inserting 3D TIFF files of processed volumes. Flow simulation was performed using the Avizo plugin XLab on segmented volumes, where four faces of the 3D data were considered to be hermetically sealed and the relevant faces labeled as inlet and outlets, with even flow distribution across the inlet plane. Flow conditions were simulated using a volume that contained the visual representation as to not restrict streamlines to within the rendered volume. Input conditions included viscosity $\left(8.9 \times 10^{-4}\right.$ Pas to approximate water) and a differential pressure from inlet to outlet of 0.1 MPa. The visual flow maps presented do not represent quantitative flow rates.

Electron microscopy using a ZEISS (USA) EVO MA10 and a JEOL (Japan) JSM-6480LV was performed at a primary accelerating voltage of $15 \mathrm{keV}$. Before imaging, samples were cut into $1 \mathrm{~mm}$ slices using a sharp scalpel for the top layer, bottom layer and a cross section before gold sputtering to coat the surface that reduces charging effects [18]. Energydispersive X-ray spectroscopy (EDS) was used to analyze the elemental composition of electron micrographs. 
Figure 1 X-ray computed tomography imaging approach. a X-ray CT imaging process, from image generation by generating $\mathrm{X}$-rays that pass through a sample and detected to produce an image. The sample is rotated around $360^{\circ}$ with 3,142 images taken that are reconstructed using backprojection mathematics and processed into a $3 \mathrm{D}$ volume with three phases. $\mathbf{b}$ Sample holder containing an isolated depth filter sheet for imaging. (a)

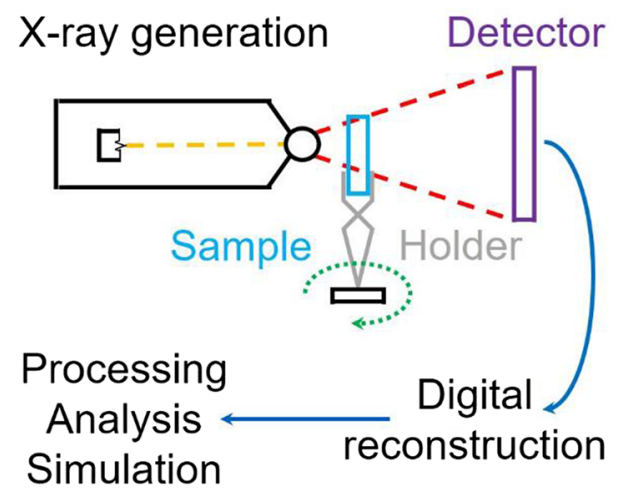

(b)

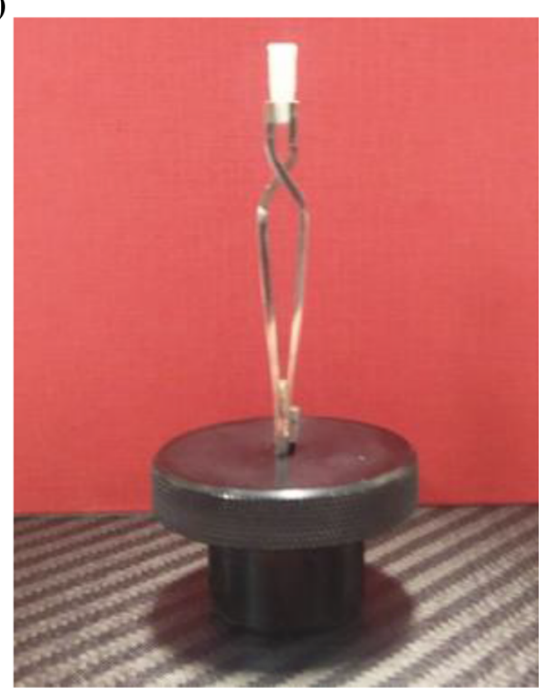

\section{Results and discussion}

\section{Imaging and void analysis}

X-ray CT was used to image the internal, three dimensional structure of depth filter materials at high resolution without the need for physical sectioning approaches that would be required for SBF or FIB tomography. Examples of this would include focused ion beam or microtomy that can provide benefits of achieving very high resolutions, but were deemed unnecessary for the purposes of this study.

The amount of sample preparation required for these imaging methods compared to X-ray CT necessitate a greater number of steps that could alter the sample prior to imaging [23]. Additionally, X-ray $\mathrm{CT}$ is non-destructive which enables iterative optimisation of imaging conditions. Figure 2 displays images at multiple length scales from a capsule to microstructure of a depth filter. Obtaining high resolutions can compromise the sample field of view, requiring stitching multiple scans together [46].

In order to achieve a pixel size sufficient to view the intricate microstructure of depth filter sheets, $3 \mathrm{~mm}$ cubes to match the media thickness were isolated and scanned. The cellulosic structure of the depth filters are fragile and so particular care was taken when producing samples for imaging because any undue changes here would detrimentally impact the representativeness of datasets going forward. Three different depth filter grades were examined in this study with varying degrees of coarseness and different nominal retention ratings. Cross-sectional images of each grade from X-ray imaging, referred to as coarse, medium and fine, are available in Fig. 3.

Even within the small filaments imaged in Fig. 3, visual differences could be observed between different grades, with the relative openness of the structure reducing from coarse to medium to fine as less black voidage space exists within each sample. Of particular interest was the presence of bright spots for all materials, suggesting an additional phase beyond the gray cellulose strands and black voidage. Triplephase tomography has been investigated in other research areas such as battery technology and so the approach was of interest here and is explored later [43]. Once the 2D images had been reconstructed into 3D volumes; then, structural analysis and simulation of flow could be performed through each depth filter structure, with examples given in Fig. 4.

The flow simulation displayed in Fig. 4a show tortuous streamlines movement through the internal depth filter geometry that feed material would experience, with tortuosity being an important factor in enabling solid-liquid separation. The lines do not represent flow rate or velocity but simply a viable path that fluid could take and the streamlines displayed are non-exhaustive of all possible flow paths.

The voidage distance maps highlighted that voids larger than the nominal retention rating were common in each grade examined that could be routinely accessed by simulated flow streamlines through structural overlay, although were not considered to be acting as channels to bypass the filter. It should be noted that under real flow the structure may undergo compression and reduce the voidage of these larger 


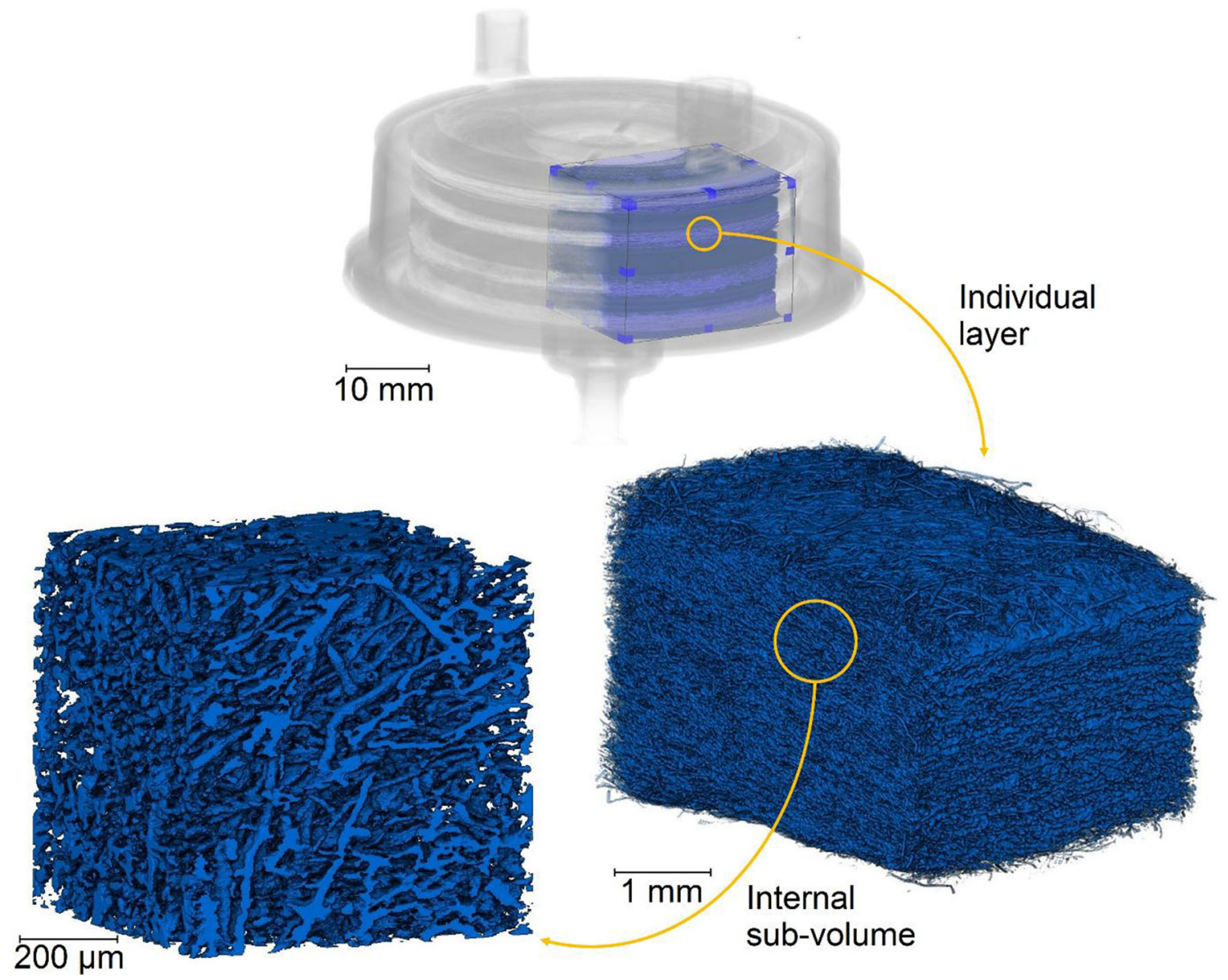

Figure 2 Multi-length scale approach to imaging depth filter materials using X-ray CT. Using the same piece of equipment, entire depth media capsules (containing 4 depth layers in this case) can be imaged right down to individual fibers within the detailed structure.

(a)

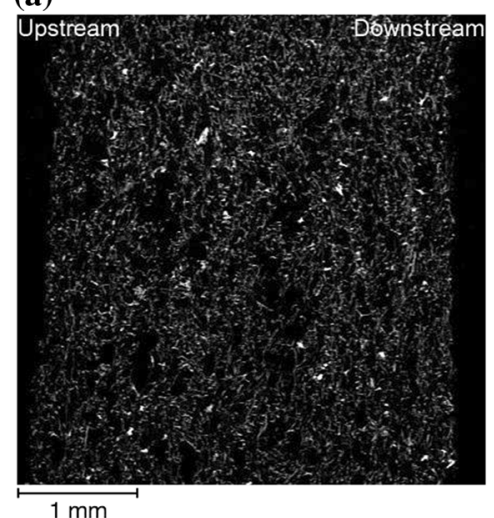

(b)

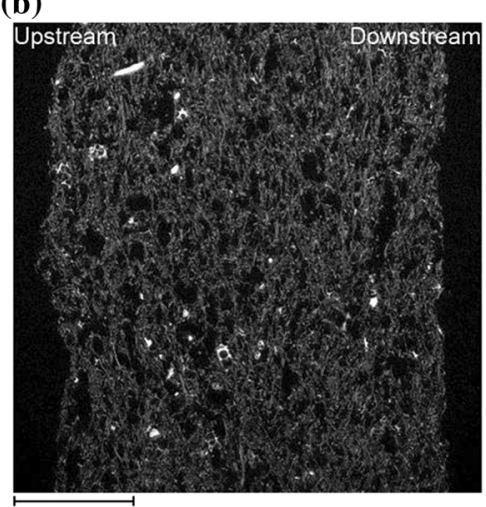

$1 \mathrm{~mm}$ (c)

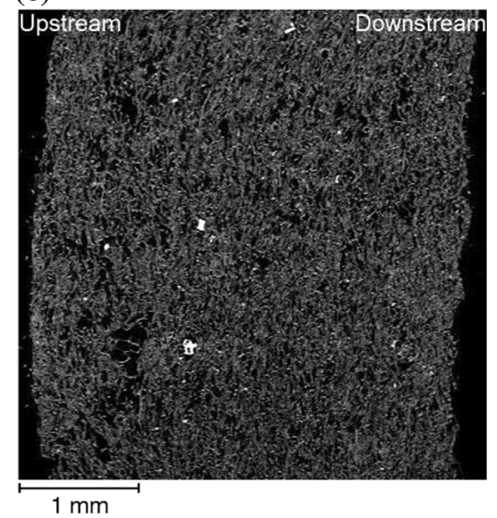

Figure 3 2D reconstructed images of depth filter material. Cross section of a Coarse, $\mathbf{b}$ Medium, c Fine depth filter grades. Further images are available in the supplementary online material.

spaces, however imaging under flow conditions at sufficient resolution was previously found to be detrimental to image quality [31].
The voidage distance maps with a cutoff at $20 \mu \mathrm{m}$ were useful for comparing the different grades by measuring the distance of voidage voxels from the nearest material voxel, with two main observations. 
(a)

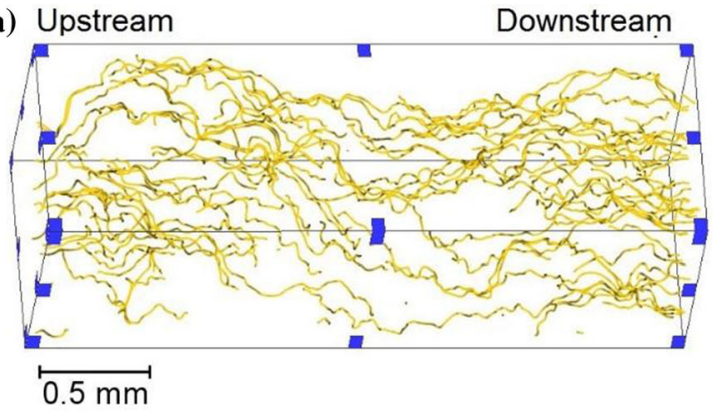

(c)

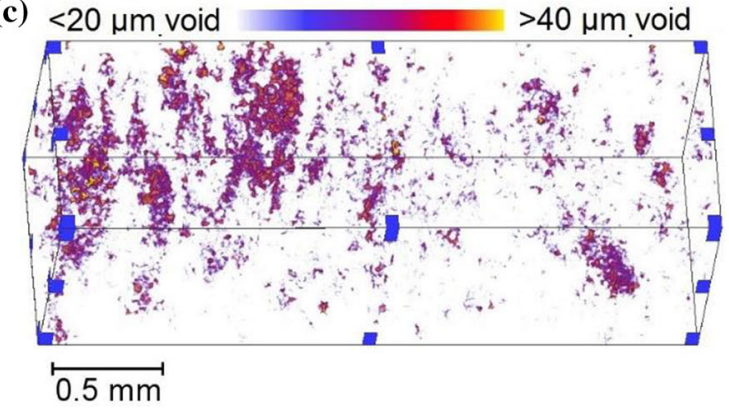

(b)

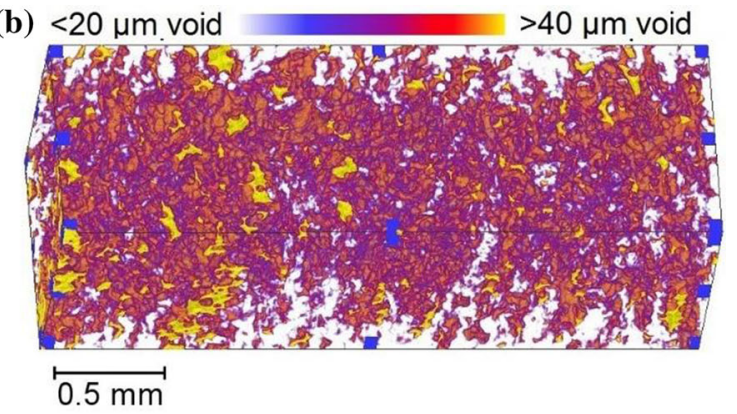

(d)

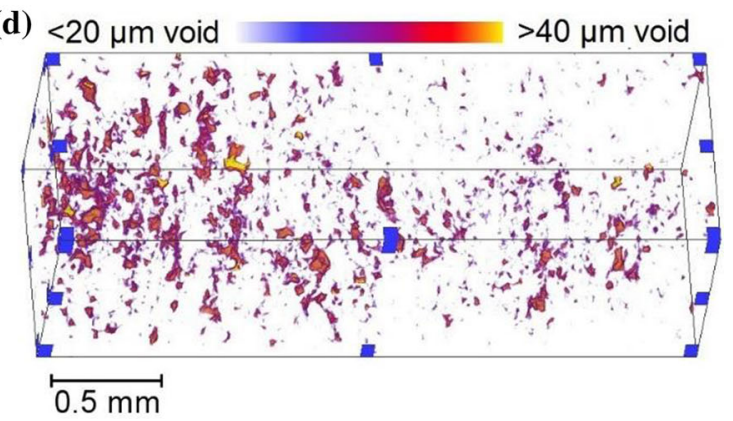

above for a coarse sub-volume. c Distance map of $20 \mu \mathrm{m}$ voids and above for a medium sub-volume. $\mathbf{d}$ Distance map of $20 \mu \mathrm{m}$ voids and above for a fine sub-volume.

sizes). This was suggested by the visual voidage distance maps and verifying that the structure changes through the material with each grade having different pore sizes $(P<5 \%$ for whole volume pore sizes tested across the three grades). Axial asymmetry would expect to reduce under flow conditions due to compression of the upper material, however the increase in material fraction closer to the downstream edge may provide structural support to the depth filter sheet as a whole.

Tortuosity factor increased from upstream to center to downstream $(P<5 \%$ for all materials-upstream, center and downstream tortuosity factor) ( $P<5 \%$ for whole volume tortuosity factor tested across the three grades). Calculation of tortuosity has developed with the increasing availability of 3D datasets and software packages that can account for structural properties which are often overlooked or oversimplified when using non-imaging approaches, however the general trend of tortuosity increasing at decreased porosities is observed here [40].

The overall average pore diameters for the medium and coarse grades were found to lie within the vendor specified nominal retention rating, suggesting that the majority of structure had been sufficiently 
Table 1 Structural measurement of depth filter void space at a $3 \mu \mathrm{m}$ pixel size

\begin{tabular}{|c|c|c|c|c|c|}
\hline Material & & Macro-porosity (\%) & Tortuosity factor & Average pore size $(\mu \mathrm{m})$ & Nominal retention $(\mu \mathrm{m})$ \\
\hline \multirow[t]{3}{*}{ Coarse } & Upstream & $75.8 \pm 1.4$ & $1.44 \pm 0.03$ & $26.3 \pm 1.9$ & \multirow[t]{3}{*}{$11-30$} \\
\hline & Center & $72.0 \pm 1.1$ & $1.53 \pm 0.05$ & $22.7 \pm 0.8$ & \\
\hline & Downstream & $71.4 \pm 1.1$ & $1.52 \pm 0.02$ & $21.5 \pm 1.0$ & \\
\hline \multirow[t]{3}{*}{ Medium } & Upstream & $58.3 \pm 0.9$ & $2.88 \pm 0.03$ & $13.2 \pm 0.4$ & \multirow[t]{3}{*}{$6-15$} \\
\hline & Center & $56.0 \pm 0.3$ & $2.90 \pm 0.08$ & $12.3 \pm 0.2$ & \\
\hline & Downstream & $52.8 \pm 1.8$ & $3.16 \pm 0.17$ & $11.1 \pm 0.6$ & \\
\hline \multirow[t]{3}{*}{ Fine } & Upstream & $48.9 \pm 1.4$ & $3.23 \pm 0.22$ & $12.1 \pm 0.5$ & \multirow[t]{3}{*}{$2-4$} \\
\hline & Center & $45.0 \pm 0.6$ & $3.63 \pm 0.16$ & $10.3 \pm 0.0$ & \\
\hline & Downstream & $41.4 \pm 0.5$ & $4.18 \pm 0.23$ & $9.3 \pm 0.0$ & \\
\hline
\end{tabular}

Results reported to three significant figures for in each case and one standard deviation. Upstream and downstream are considered to be the region up to $25 \%$ away from the respective filter edge, with center being the remaining $50 \%$

resolved. However, for the fine depth filter, the average pore size was found to be above the nominal retention rating, indicating that the finest pores and features are not imaged using a $3 \mu \mathrm{m}$ pixel size, or boundaries between phases were not adequately defined. Nominal retention rating is typically evaluated empirically using a model system and does not directly measure pore size as can be achieved when using 3D datasets acquired here.

The depth filter samples examined in this study were dry and unused which was necessary for imaging at microscale resolutions using $\mathrm{X}$-ray $\mathrm{CT}$, a previous study had found that imaging fouled bioprocessing samples severely compromised image quality [31]. If the depth filter had been used and could be imaged to view the internal features, then it would be expected that the upstream surface that contains the largest porous spaces would compress the most.

Scaled up depth filtration systems would be expected to use the same type of depth media as seen in the capsule displayed in Fig. 2, however imaging larger devices compromises achievable resolution and so would not be appropriate for this study when observing microscale features. Depth filter sheets can be delicate and so ensuring structural integrity and minimizing media pore size variability are two key considerations when scaling up.

\section{Pixel size improvements}

In addition to the pore size to nominal retention rating disparity, porosity measurements below 50\% were unlikely to be representative because depth filters rely on a large voidage and therefore holding capacity. Improvements to pixel size were required in order to identify the finest features within each sample and enable more accurate measurement of voidage and material phases. Two approaches were taken, the first using 'transmission' mode on the same $X$-ray CT equipment in order to improve pixel size from $3 \mu \mathrm{m}$ to $2 \mu \mathrm{m}$ and the second being the use of an alternative piece of equipment at $1 \mu \mathrm{m}$ and $0.5 \mu \mathrm{m}$ pixel sizes.

In order to effectively image at an improved resolution, re-optimisation of other X-ray $\mathrm{CT}$ imaging settings was required to account for switching to a tungsten target from silver for X-ray generation. A tungsten target was the only available source metal for X-ray generation when using the higher resolution modes and so adjustment of other settings such as primary acceleration voltage was required. Initially a silver target had been found to be better suited for generating images of the cellulosic samples, however it was deemed to be more appropriate for direct comparison between samples if key imaging setup factors including the type of metal used in the target were consistent between pixel sizes [29].

With these optimized settings, Fig. 5 displays an example image at the same position for the fine grade at $3 \mu \mathrm{m}, 2 \mu \mathrm{m}$ and $1 \mu \mathrm{m}$ pixel sizes, with the improved resolution imaging necessitating internal tomography. A $0.5 \mu \mathrm{m}$ pixel size variant could only produce very small volumes, which also required very long scan times and so was not taken forward for analysis but included in the Supplementary online material for Fig. 5. 


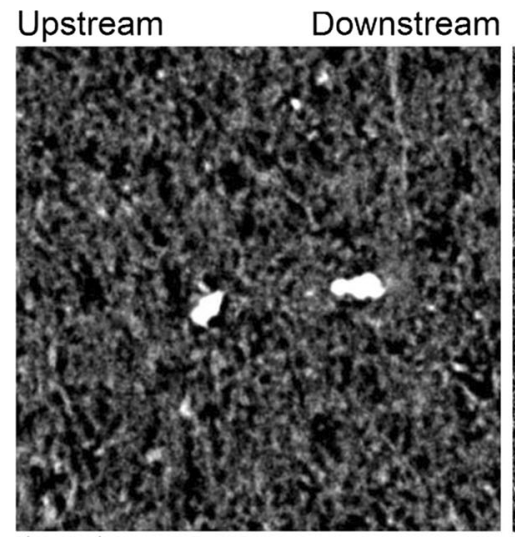

$2 \stackrel{200 \mu \mathrm{m}}{1}$
$3 \mu \mathrm{m}$ pixel size

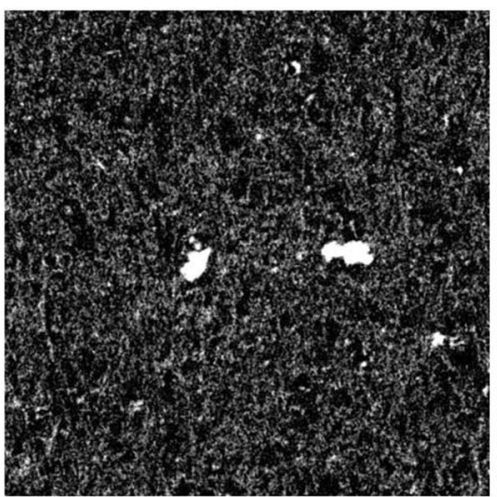

$2 \mu \mathrm{m}$ pixel size

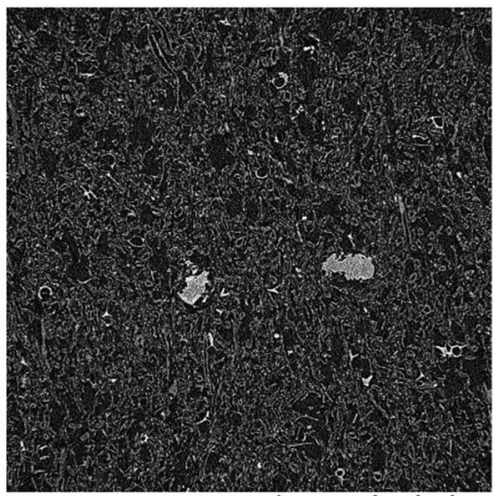

$1 \mu \mathrm{m}$ pixel size

Figure 5 Comparison of 2D images from X-ray CT acquisition of a fine grade at different pixel sizes at the same coordinates. Further examples for all depth filter grades are available in the supplementary online material.

It can be seen that the amount of detail resolved for the fine depth filter grade increases as the pixel size improves, with the medium and coarse variants presented in the Supplementary online material for Fig. 5 also improving but to a lesser extent, which was expected due to majority of structure already being resolved and measured in Table 1. A key tradeoff to be considered when applying high resolution imaging in this case was that the $1 \mu \mathrm{m}$ pixel datasets did not cover the entire thickness of the sample and so measuring filter asymmetry as a function of distance through the sample was not performed that was possible for the $3 \mu \mathrm{m}$ pixel size data [46]. The corresponding calculations from the 3D imaging for the fine depth filter grade are presented in Table 2.

It was found that at an improved pixel size as presented in Table 2 a greater degree of porosity could be identified compared to lower resolutions. This suggested that finer voidage-material boundaries were being visualized and characterized which were previously solely attributed to the material phase using the $3 \mu \mathrm{m}$ pixel size measurements. This coincided with decreases to both average pore size and simulated tortuosity factor as the smaller gaps provided more avenues for flow, highlighting the need to match resolution to expected feature sizes.

Tortuosity reduced when improving pixel size, suggesting that micropores contribute to tortuosity of potential flow paths through depth filters. Porosity measurements for all grades was obtained at $70 \%$ to $80 \%$, with a large voidage space required for depth filters to provide holding capacity. Results for the coarse and medium grades for each of the pixel sizes investigated are available in the Supplementary online material for Table 2.

Nominal retention ratings of these filters are not directly defined in terms of pore size and is instead a threshold relating to transmission of a standard particle size. Therefore, a nominal retention rating does not account for the large vacuous spaces that are measured using a tomographic approach. This results in the calculations performed here to be potentially above the stated nominal retention rating for the fine grade even when applying a $1 \mu \mathrm{m}$ pixel size.

\section{Triple-phase tomography}

In each of the grades, the material phase had a combination of dark and bright areas, indicating that

Table 2 Comparison between different pixel sizes achieved for imaging and measuring fine grade depth filter structure

\begin{tabular}{llllr}
\hline Pixel size $(\mu \mathrm{m})$ & Thickness imaged $(\mathrm{mm})$ & Porosity $(\%)$ & Tortuosity factor & Average pore size $(\mu \mathrm{m})$ \\
\hline 3 & $3+$ & $43.8 \pm 4.6$ & $3.87 \pm 1.01$ & $11.5 \pm 1.3$ \\
2 & $3+$ & $64.7 \pm 3.0$ & $1.90 \pm 0.17$ & $9.3 \pm 1.0$ \\
1 & 1.5 & $71.5 \pm 1.0$ & $1.43 \pm 0.04$ & $7.3 \pm 0.4$ \\
\hline
\end{tabular}

Results reported to three significant figures in each case and one standard deviation 
(a)

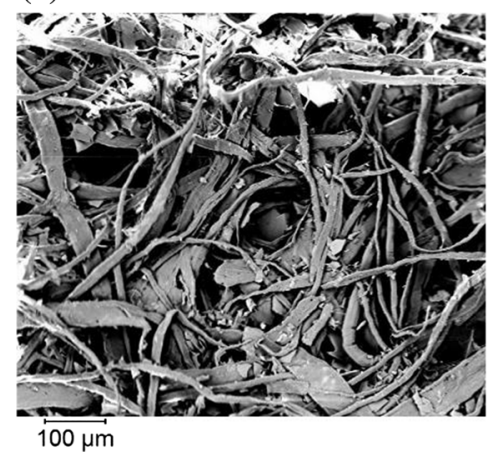

(b)



(c)

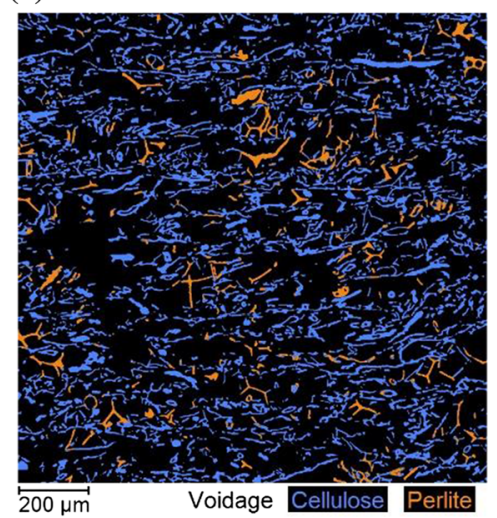

Figure 6 Triple-phase tomographic segmentation of a coarse depth filter grade. a Electron micrograph of downstream surface, displaying cellulose fibers and perlite shards, b 2D internal slice coarse grade before segmentation. c 2D internal coarse grade slice

more than one component was present within the samples. Many depth filters including these are constituted of a cellulose base but also contain perlite, or in some cases diatomaceous earth. Electron microscopy and EDS were used to image the surface of these depth filters and identify the elemental composition, with an example available in Fig. 6a. In the electron micrograph both cellulose fibers and smaller perlite shards can be seen on the downstream side of a coarse grade, with EDS results indicated that the shards have high silicon and oxygen content that are the major elemental components of perlite.

The presence of multiple phases within each grade raises questions concerning the relative quantities of these constituent materials. By having high-resolution images, then analysis of these complex structures can be performed through digital processing and characterization of each grade. The regular presence of perlite in the structure resulted in individual segmentation of cellulose and perlite for each grade. $1 \mu \mathrm{m}$ pixel size datasets were used because they captured the finer features of each sample, enabling smaller shards to be identified.

Figure $6 \mathrm{~b}$ and c display a 2D slice before and after triple-phase segmentation of voidage, cellulose and perlite for the coarse depth filter grade, with corresponding triple-phase segmentation images for the medium and fine grades available in the online supplementary material for Fig. 6 . The 2D slices were rendered into 3D sub-volumes, as seen in Fig. 7 with a thinner cross section displayed in the online supplementary material for Fig. 7. digitally segmented into black voidage, blue cellulose and orange perlite. Examples of segmentation for the other grades are available in the supplementary online material.

Similar to the 2D slices, the bright space in the 3D renders for each grade can be seen as orange perlite in the triple-phase representations, with 3D rendering being useful for gaining a greater spatial appreciation as to how these two components are distributed within the filter. By separating perlite and cellulose the relative proportion by volume can be measured. Throughout the images displayed in Fig. 2 and the 3D renders in Fig. 7 the order of perlite quantities visually align with the measurements in Table 3. Approximately $15 \%$ perlite was present in the material phase for the coarse grade, $23 \%$ for the medium grade but less than $2 \%$ for the fine variant.

In the case of the smallest features, for example isolated perlite material identified as a single or small group of pixels, some degree of erroneous segmentation into the incorrect phase was encountered even when digital processing had improved the signal to noise ratio. Therefore, the perlite fraction may be greater than measured in these datasets, particularly for the finer grade and would be of interest to explore further in future studies.

\section{Conclusions}

By applying a non-invasive and non-destructive tomographic approach, the detailed 3D internal structure of various depth filter materials could be imaged in high resolution to evaluate important geometrical aspects such as porosity, tortuosity and pore size without need for physical sectioning beyond preparative sample isolation. Obtaining a 
(a)

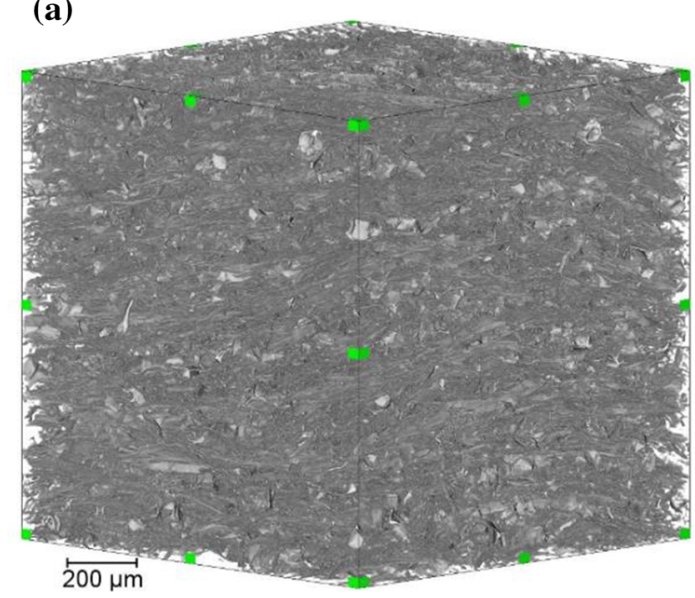

(c)

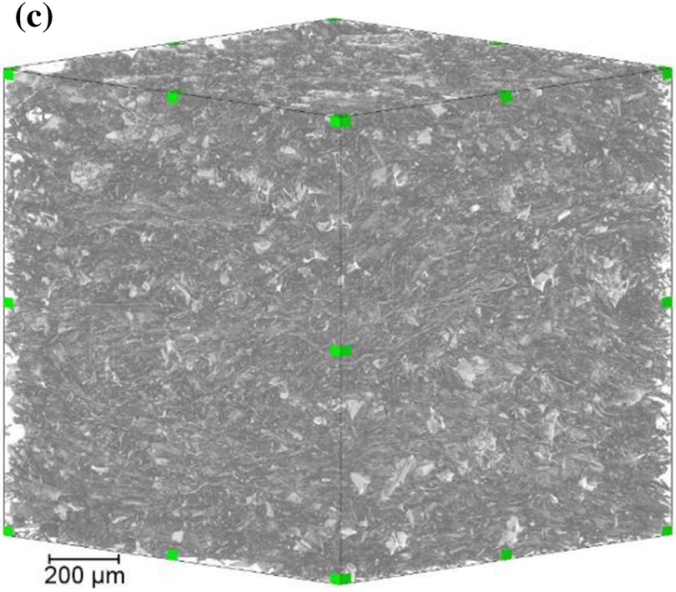

(e)

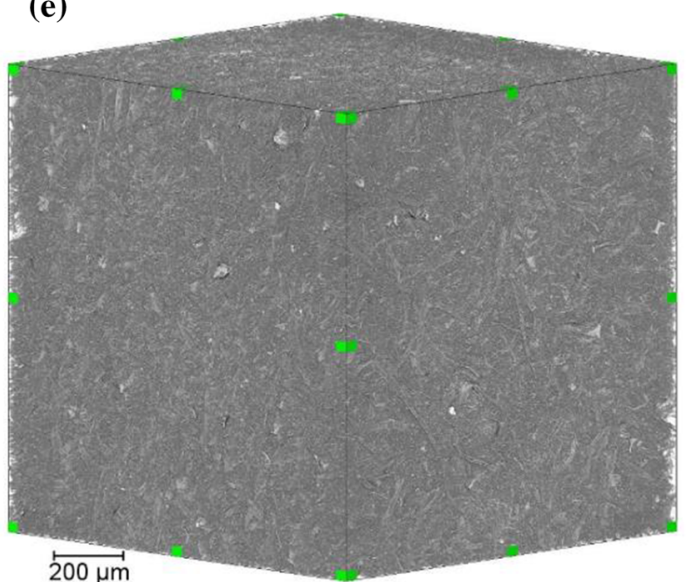

Figure 7 3D renders of depth filter grade internal sub-volumes. a Coarse grade before segmentation. b Coarse grade after segmentation into transparent voidage, blue cellulose and orange

pixel size below the nominal retention rating was found to be important, although outright resolution often brings drawbacks, for example field of view (b)

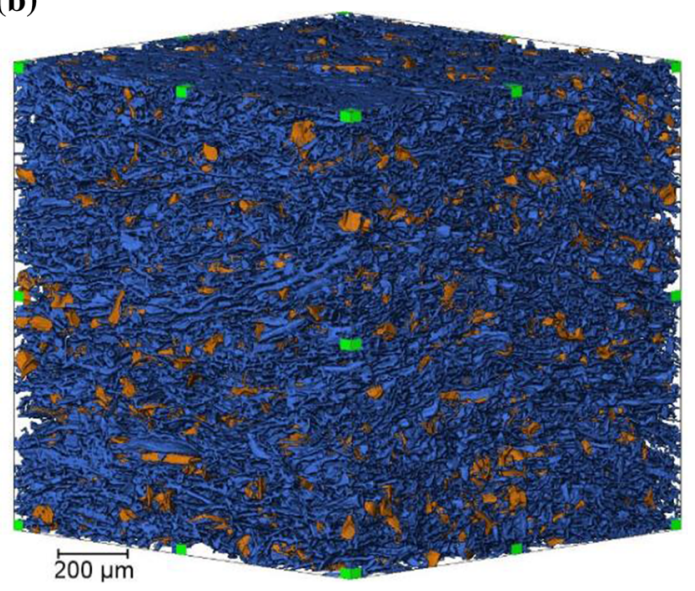

(d)

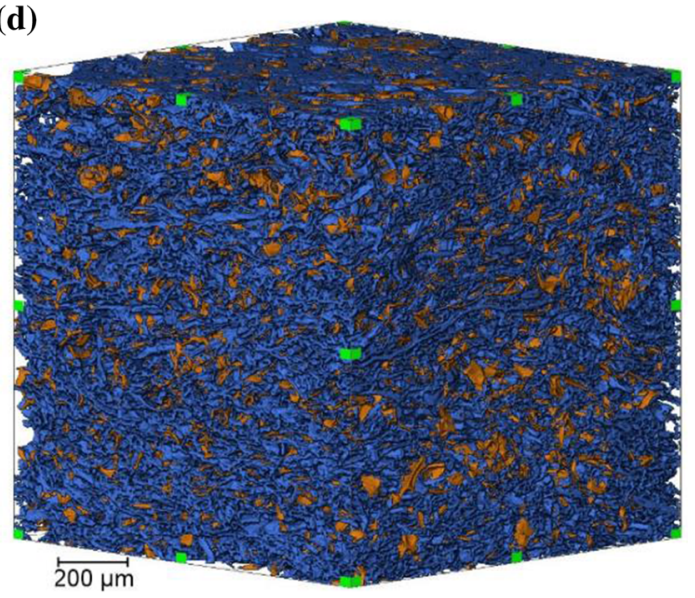

(f)

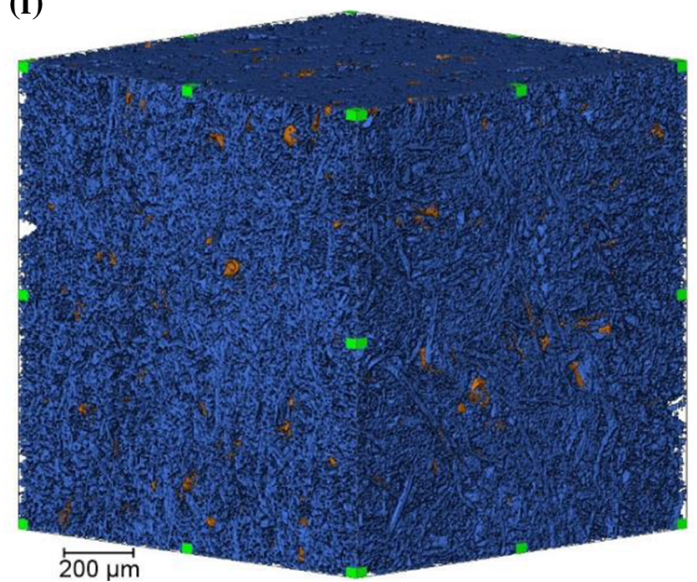

perlite. c Medium grade before segmentation. $\mathbf{d}$ Medium grade following segmentation. e Fine grade before segmentation. f Fine grade following segmentation.

limitations and so determining the most suitable pixel size for 3D imaging may require experimental comparison. Without a sufficient resolution, 
Table 3 Triple-phase tomography measurements of depth filter components

\begin{tabular}{llll}
\hline Volume (\%) & Coarse & Medium & Fine \\
\hline Perlite & 3 & 6 & 1 \\
Cellulose & 18 & 18 & 28 \\
Air & 79 & 76 & 71 \\
\hline
\end{tabular}

then key structural data will be lost that will reduce the representativeness of digital measurements, as was observed in this study by comparing pixel sizes when imaging the same location within a sample.

By undertaking a multi-length scale and triplephase tomographic approach then a more comprehensive understanding of how depth filter microstructure relates to function was obtained. Positional based analysis of each constituent from upstream to downstream surface identified a change in voidage and pore sizes. For future studies it would also be of interest to incorporate diatomaceous earth as a fourth phase for relevant depth filters.

Examining these filters and how they change under typical operational conditions would be useful for building a more comprehensive understanding of bioprocess material behavior. This would involve implementing complementary imaging techniques, for example confocal laser scanning microscopy in order to maximize the amount of visual information that could be gathered for measurement and evaluation. Using real bioprocessing structures from 3D imaging is of interest for future studies involving computational fluid dynamics to further relate filter structure to function and performance. A computational approach may allow for operating conditions optimization and the potential for design suggestions to be made for new depth filter media.

\section{Acknowledgements}

This work was funded by the University College London - Pall Biotech Centre of Excellence and the Engineering and Physical Sciences Research Council (EPSRC) Grants EP/S021868/1 and EP/L01520X/1. Paul Shearing acknowledges support from the Royal Academy of Engineering. We would like to express our gratitude to the support and guidance provided by Mike Collins, Dave Hayden, Nigel Jackson,
Ruediger Leibnitz and Kalliopi Zourna at Pall Biotech.

\section{Declarations}

Conflict of interest The authors declare that they have no conflict of interest.

Supplementary Information: The online version contains supplementary material available at http s://doi.org/10.1007/s10853-021-06238-w.

Open Access This article is licensed under a Creative Commons Attribution 4.0 International License, which permits use, sharing, adaptation, distribution and reproduction in any medium or format, as long as you give appropriate credit to the original author(s) and the source, provide a link to the Creative Commons licence, and indicate if changes were made. The images or other third party material in this article are included in the article's Creative Commons licence, unless indicated otherwise in a credit line to the material. If material is not included in the article's Creative Commons licence and your intended use is not permitted by statutory regulation or exceeds the permitted use, you will need to obtain permission directly from the copyright holder. To view a copy of this licence, visit http://creativecommons.org/licen ses/by/4.0/.

\section{References}

[1.] Gronemeyer P, Ditz R, Strube J (2014) Trends in upstream and downstream process development for antibody manufacturing. Bioengineering 1:188-212. https://doi.org/10.33 90/bioengineering 1040188

[2.] Liu HF, Ma J, Winter C, Bayer R (2010) Recovery and purification process development for monoclonal antibody production. MAbs 2:480-499. https://doi.org/10.4161/mabs. 2.5.12645

[3.] Nguyen HC, Langland AL, Amara JP, Dullen M, Kahn DS, Costanzo JA (2018) Improved HCP reduction using a new, all-synthetic depth filtration media within an antibody purification process. Biotechnol J 14:1-11. https://doi.org/ 10.1002/biot.201700771

[4.] Goldrick S, Joseph A, Mollet M, Turner R, Gruber D, Farid SS, Titchener-Hooker NJ (2017) Predicting performance of constant flow depth filtration using constant pressure filtration data. J Memb Sci 531:138-147. https://doi.org/10.1016/ j.memsci.2017.03.002 
[5.] Krupp AU, Please CP, Kumar A, Griffiths IM (2017) Scaling-up of multi-capsule depth filtration systems by modeling flow and pressure distribution. Sep Purif Technol 172:350-356. https://doi.org/10.1016/j.seppur.2016.07.028

[6.] Zydney AL (2016) Continuous downstream processing for high value biological products: a review. Biotechnol Bioeng 113:465-475. https://doi.org/10.1002/bit.25695

[7.] Noyes A, Basha J, Frostad J, Cook S, Millard D, Mullin J, LaCasse D, Wright RS, Huffman B, Fahrner R, Godavarti R, Titchener-Hooker NJ, Sunasara K, Mukhopadhyay T (2015) A modular approach for the ultra-scale-down of depth filtration. J Memb Sci 496:199-210. https://doi.org/ 10.1016/j.memsci.2015.07.010

[8.] Tait AS, Tarrant RDR, Velez-Suberbie ML, Spencer DIR, Bracewell DG (2013) Differential response in downstream processing of $\mathrm{CHO}$ cells grown under mild hypothermic conditions. Biotechnol Prog 29:688-696. https://doi.org/10. 1002/btpr.1726

[9.] Ziel R, Haus A, Tulke A (2008) Quantification of the pore size distribution ( porosity profiles ) in microfiltration membranes by SEM, TEM and computer image analysis. J Memb Sci 323:241-246. https://doi.org/10.1016/j.memsci. 2008.05.057

[10.] Nejatishahidein N, Borujeni EE, Roush DJ, Zydney AL (2020) Effectiveness of host cell protein removal using depth filtration with a filter containing diatomaceous earth. Biotechnol Prog. https://doi.org/10.1002/btpr.3028

[11.] Khanal O, Singh N, Traylor SJ, Xu X, Ghose S, Li ZJ, Lenhoff AM (2018) Contributions of depth filter components to protein adsorption in bioprocessing. Biotechnol Bioeng 115:1938-1948. https://doi.org/10.1002/bit.26707

[12.] Singh N, Arunkumar A, Chollangi S, Tan ZG, Borys M, Li ZJ (2016) Clarification technologies for monoclonal antibody manufacturing processes: current state and future perspectives. Biotechnol Bioeng 113:698-716. https://doi. org/10.1002/bit.25810

[13.] Cooper SJ, Kishimoto M, Tariq F, Bradley RS, Marquis AJ, Brandon NP, Kilner JA, Shearing PR (2013) Microstructural analysis of an LSCF cathode using in situ tomography and simulation. ECS Trans 57:2671-2678. https://doi.org/10.11 49/05701.2671 ecst

[14.] Heenan TMM, Finegan DP, Tjaden B, Iacoviello F, Millichamp J, Brett DJL, Shearing PR (2018) 4D nano-tomography of electrochemical energy devices using labbased X-ray imaging. Nano Energy 47:556-565. https://doi. org/10.1016/j.nanoen.2018.03.001

[15.] Withers PJ (2007) X-ray nanotomography. Mater Today 10:26-34. https://doi.org/10.1016/S1369-7021(07)70305-X

[16.] Mertens JCE, Henderson K, Cordes NL, Pacheco R, Xiao X, Williams JJ, Chawla N, Patterson BM (2017) Analysis of thermal history effects on mechanical anisotropy of 3Dprinted polymer matrix composites via in situ X-ray tomography. J Mater Sci 52:12185-12206. https://doi.org/ 10.1007/s10853-017-1339-4

[17.] Chapelle L, Lyckegaard A, Kusano Y, Gundlach C, Foldschak MR, Lybye D, Brøndsted P (2018) Determination of the fibre orientation distribution of a mineral wool network and prediction of its transverse stiffness using X-ray tomography. J Mater Sci 53:6390-6402. https://doi.org/10. 1007/s10853-018-2044-7

[18.] Denk W, Horstmann H (2004) Serial block-face scanning electron microscopy to reconstruct three-dimensional tissue nanostructure. PLoS Biol 2:1900-1909. https://doi.org/10. 1371/journal.pbio.0020329

[19.] Jungreuthmayer C, Steppert P, Sekot G, Zankel A, Reingruber H, Zanghellini J, Jungbauer A (2015) The 3D pore structure and fluid dynamics simulation of macroporous monoliths: high permeability due to alternating channel width. J Chromatogr A 1425:141-149. https://doi.org/10. 1016/j.chroma.2015.11.026

[20.] Müllner T, Zankel A, Höltzel A, Svec F, Tallarek U (2017) Morphological properties of methacrylate-based polymer monoliths: from gel porosity to macroscopic inhomogeneities. Langmuir 33:2205-2214. https://doi.org/10.1021 /acs.langmuir.7b00337

[21.] Bailey JJ, Heenan TMM, Finegan DP, Lu X, Daemi DR, Iacoviello F, Backeberg NR, Taiwo OO, Brett DJL, Atkinson A, Shearing PR (2017) Laser-preparation of geometrically optimised samples for X-ray nano-CT. J Microsc 267:384-396. https://doi.org/10.1111/jmi.12577

[22.] Shearing PR, Golbert J, Chater RJ, Brandon NP (2009) 3D reconstruction of SOFC anodes using a focused ion beam lift-out technique. Chem Eng Sci 64:3928-3933. https://doi. org/10.1016/j.ces.2009.05.038

[23.] Zankel A, Wagner J, Poelt P (2014) Serial sectioning methods for 3D investigations in materials science. Micron 62:66-78. https://doi.org/10.1016/j.micron.2014.03.002

[24.] Burnett TL, McDonald SA, Gholinia A, Geurts R, Janus M, Slater T, Haigh SJ, Ornek C, Almuaili F, Engelberg DL, Thompson GE, Withers PJ (2014) Correlative tomography. Sci Rep 4:1-6. https://doi.org/10.1038/srep04711

[25.] Izzo JR, Joshi AS, Grew KN, Chiu WKS, Tkachuk A, Wang $\mathrm{SH}$, Yun W (2008) Nondestructive reconstruction and analysis of SOFC anodes using X-ray computed tomography at sub-50 $\mathrm{nm}$ resolution. $\mathrm{J}$ Electrochem Soc 155:504-508. https://doi.org/10.1149/1.2895067

[26.] Shearing PR, Howard LE, Jørgensen PS, Brandon NP, Harris SJ (2010) Characterization of the 3-dimensional microstructure of a graphite negative electrode from a Li-ion 
battery. Electrochem commun 12:374-377. https://doi.org/ 10.1016/j.elecom.2009.12.038

[27.] Tariq F, Yufit V, Kishimoto M, Shearing PR, Menkin S, Golodnitsky D, Gelb J, Peled E, Brandon NP (2014) Threedimensional high resolution X-ray imaging and quantification of lithium ion battery mesocarbon microbead anodes. J Power Sources 248:1014-1020. https://doi.org/10.1016/j. jpowsour.2013.08.147

[28.] Angelo JM, Cvetkovic A, Gantier R, Lenhoff AM (2013) Characterization of cross-linked cellulosic ion-exchange adsorbents: 1 structural properties. J Chromatogr A 1319:46-56. https://doi.org/10.1016/j.chroma.2013.10.003

[29.] Johnson TF, Levison PR, Shearing PR, Bracewell DG (2017) X-ray computed tomography of packed bed chromatography columns for three dimensional imaging and analysis. J Chromatogr A 1487:108-115. https://doi.org/10. 1016/j.chroma.2017.01.013

[30.] Johnson TF, Bailey JJ, Iacoviello F, Welsh JH, Levison PR, Shearing PR, Bracewell DG (2018) Three dimensional characterisation of chromatography bead internal structure using X-ray computed tomography and focused ion beam microscopy. J Chromatogr A 1566:79-88. https://doi.org/ 10.1016/j.chroma.2018.06.054

[31.] Johnson TF, Iacoviello F, Hayden DJ, Welsh JH, Levison PR, Shearing PR, Bracewell DG (2020) Packed bed compression visualisation and flow simulation using an erosiondilation approach. J Chromatogr A 1611:1-9. https://doi. org/10.1016/j.chroma.2019.460601

[32.] Wankmüller F, Meffert M, Russner N, Weber A, Schmieg J, Störmer H, Dickel T, Lupetin P, Maier N, Gerthsen D, IversTiffée E (2020) Multi-scale characterization of ceramic inert-substrate-supported and co-sintered solid oxide fuel cells. J Mater Sci 55:11120-11136. https://doi.org/10.1007/ s10853-020-04873-3

[33.] Finegan DP, Darcy E, Keyser M, Tjaden B, Heenan TMM, Jervis R, Bailey JJ, Vo NT, Magdysyuk OV, Drakopoulos M, Di Michiel M, Rack A, Hinds H, Brett DJL, Shearing PR (2018) Identifying the cause of rupture of Li-Ion batteries during thermal runaway. Adv Sci 5:1-13. https://doi.org/10. 1002/advs.201700369

[34.] Heenan TMM, Tan C, Jervis R, Lu X, Brett DJL, Shearing PR (2019) Representative resolution analysis for X-ray CT: a solid oxide fuel cell case study. Chem Eng Sci X 4:1-9. h ttps://doi.org/10.1016/j.cesx.2019.100043

[35.] Robinson JB, Brown LD, Jervis R, Taiwo OO, Heenan TMM, Millichamp J, Mason TJ, Neville TP, Clague R, Eastwood DS, Reinhard C, Lee PD, Brett DJL, Shearing PR (2015) Investigating the effect of thermal gradients on stress in solid oxide fuel cell anodes using combined synchrotron radiation and thermal imaging. $\mathrm{J}$ Power Sources
288:473-481. https://doi.org/10.1016/j.jpowsour.2015.04. 104

[36.] Dolamore F, Fee C, Dimartino S (2018) Modelling ordered packed beds of spheres: the importance of bed orientation and the influence of tortuosity on dispersion. J Chromatogr A 1532:150-160. https://doi.org/10.1016/j.chroma.2017.12. 004

[37.] Tjaden B, Lane J, Neville TP, Brown LD, Mason TJ, Tan C, Lounasvuori MM, Brett DJL, Shearing PR (2017) Comparison of ionic and diffusive mass transport resistance in porous structures. ECS Trans 75:135-145. https://doi.org/ $10.1149 / 07542.0135$ ecst

[38.] Kehrwald D, Shearing PR, Brandon NP, Sinha PK, Harris SJ (2011) Local tortuosity inhomogeneities in a lithium battery composite electrode. J Electrochem Soc 158:A1393. https://doi.org/10.1149/2.079112jes

[39.] Cooper SJ, Bertei A, Shearing PR, Kilner JA, Brandon NP (2016) TauFactor: an open-source application for calculating tortuosity factors from tomographic data. SoftwareX 5:203-210. https://doi.org/10.1016/j.softx.2016.09.002

[40.] Tjaden B, Cooper SJ, Brett DJ, Kramer D, Shearing PR (2016) On the origin and application of the Bruggeman correlation for analysing transport phenomena in electrochemical systems. Curr Opin Chem Eng 12:44-51. https://d oi.org/10.1016/j.coche.2016.02.006

[41.] Tjaden B, Brett DJL, Shearing PR (2018) Tortuosity in electrochemical devices: a review of calculation approaches. Int Mater Rev 63:47-67. https://doi.org/10.1080/09506608. 2016.1249995

[42.] Iacoviello F, Lu X, Mitchell TM, Brett DJL, Shearing PR (2019) The imaging resolution and Knudsen effect on the mass transport of shale gas assisted by multi-length scale X-ray computed tomography. Sci Rep 9:1-10. https://doi. org/10.1038/s41598-019-55999-7

[43.] Lu X, Taiwo OO, Bertei A, Li T, Li K, Brett DJL, Shearing PR (2017) Multi-length scale tomography for the determination and optimization of the effective microstructural properties in novel hierarchical solid oxide fuel cell anodes. J Power Sources 367:177-186. https://doi.org/10.1016/j.jp owsour.2017.09.017

[44.] Meyer Q, Hack J, Mansor N, Iacoviello F, Bailey JJ, Shearing PR, Brett DJL (2019) Multi-scale imaging of polymer electrolyte fuel cells using X-ray micro- and nanocomputed tomography, transmission electron microscopy and helium-ion microscopy. Fuel Cells 19:35-42. https://d oi.org/10.1002/fuce.201800047

[45.] Lu X, Heenan TMM, Bailey JJ, Li T, Li K, Brett DJL, Shearing PR (2017) Correlation between triple phase boundary and the microstructure of solid oxide fuel cell anodes: the role of composition, porosity and $\mathrm{Ni}$ 
densification. J Power Sources 365:210-219. https://doi.org/ 10.1016/j.jpowsour.2017.08.095

[46.] Maire E, Withers PJ (2013) Quantitative X-ray tomography. Int Mater Rev 59:1-43. https://doi.org/10.1179/174328041 3y.0000000023
Publisher's Note Springer Nature remains neutral with regard to jurisdictional claims in published maps and institutional affiliations. 